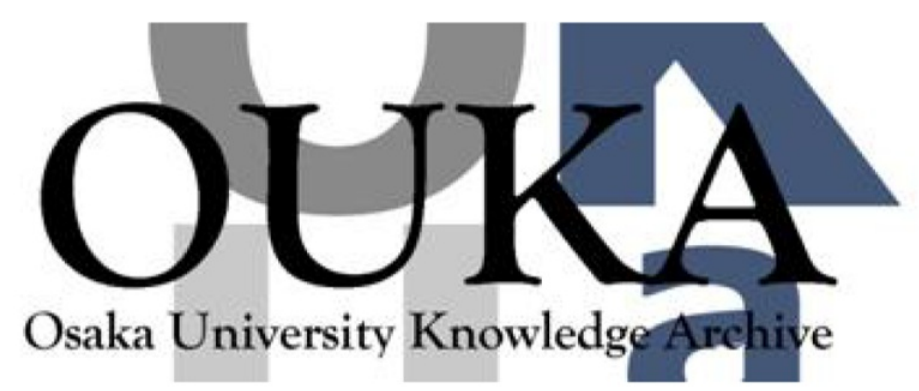

\begin{tabular}{|c|l|}
\hline Title & Stop-and-go kinetics in amyloid fibrillation \\
\hline Author(s) & $\begin{array}{l}\text { Ferkinghoff-Borg, Jesper; Fonslet, Jesper; } \\
\text { Andersen, Christian Beyschau et al. }\end{array}$ \\
\hline Citation & Physical Review E. 80 p. 010901 \\
\hline Issue Date & $2010-07$ \\
\hline oaire:version & VoR \\
\hline URL & https://hdl. handle. net/11094/71262 \\
\hline rights & ○ 2010 The American Physical Society \\
\hline Note & \\
\hline
\end{tabular}

Osaka University Knowledge Archive : OUKA

https://ir. Library. osaka-u. ac. jp/

Osaka University 


\title{
Stop-and-go kinetics in amyloid fibrillation
}

\author{
Jesper Ferkinghoff-Borg, ${ }^{1, *}$ Jesper Fonslet, ${ }^{2,3}$ Christian Beyschau Andersen, ${ }^{4,5}$ Sandeep Krishna, ${ }^{2}$ Simone Pigolotti, ${ }^{2}$ \\ Hisashi Yagi, ${ }^{6,7}$ Yuji Goto, ${ }^{6,7}$ Daniel Otzen, ${ }^{8}$ and Mogens H. Jensen ${ }^{2, \dagger}$ \\ ${ }^{1}$ DTU Elektro, Building 349, Ørsteds Plads, Technical University of Denmark, 2800 Lyngby, Denmark \\ ${ }^{2}$ Niels Bohr Institute, Blegdamsvej 17, DK-2100 Copenhagen, Denmark \\ ${ }^{3}$ Herlev Hospital, Klinisk Fysiologisk Afdeling, Herlev Ringvej 75, DK-2730 Herlev, Denmark \\ ${ }^{4}$ Novo Nordisk A/S, Protein Structure and Biophysics, Novo Nordisk Park, DK-2760 Målov, Denmark \\ ${ }^{5}$ National Research Council, Institute of Biophysics, Via Ugo La Malfa 153, I-90146 Palermo, Italy \\ ${ }^{6}$ Osaka University, Institute for Protein Research, Yamadaoka 3-2, Suita, Osaka 565-0871, Japan \\ ${ }^{7}$ CREST, Japan Science and Technology Agency, Saitama, Japan \\ ${ }^{8}$ Department of Molecular Biology, Århus University, Gustav Wieds Vej 10 C, 8000 Århus C, Denmark \\ (Received 13 October 2009; revised manuscript received 4 May 2010; published 1 July 2010)
}

\begin{abstract}
Many human diseases are associated with protein aggregation and fibrillation. We present experiments on in vitro glucagon fibrillation using total internal reflection fluorescence microscopy, providing real-time measurements of single-fibril growth. We find that amyloid fibrils grow in an intermittent fashion, with periods of growth followed by long pauses. The observed exponential distributions of stop and growth times support a Markovian model, in which fibrils shift between the two states with specific rates. Even if the individual rates vary considerably, we observe that the probability of being in the growing (stopping) state is very close to $1 / 4$ (3/4) in all experiments.
\end{abstract}

DOI: 10.1103/PhysRevE.82.010901

PACS number(s): 87.14.em, 82.39.-k, 87.15.bk

Protein fibrillation is the process by which misfolded proteins tend to form large linear aggregates [1]. Its importance is related to the role played in many degenerative diseases, such as Parkinson, Alzheimer, Huntington, and prion diseases [2]. While our knowledge of the structural properties of these fibrils improves at great pace [3-5], the dynamics of their growth process is still poorly understood. The formation of amyloid fibrils involves at least two steps: the formation of growth centers by primary nucleation, which is often a slow process, followed by elongation through addition of monomers [6]. In many cases, a so-called secondary nucleation mechanism is also involved, whereby new growth centers are formed from existing fibrils [7-11]. Whereas the process of secondary nucleation is known to entail a number of different mechanisms [11], the primary elongation process has not been elucidated to the same level of detail.

In this Rapid Communication, we present an experimental and theoretical study of the elongation process of glucagon fibrils. Glucagon is a small peptide hormone consisting of only 29 amino acids produced in the pancreas. It has the opposite effect to that of insulin and therefore increases blood glucose levels when released. As a model system for protein fibrillation, glucagon kinetics has provided insights into the early oligomerization stages of the process [12-14], the interplay between growth and fibril morphology $[15,16]$, and amyloid branching [11]. Here, we focus on the properties of the late-stage elongation process.

Experiments were performed on samples of glucagon monomers in solution. In order to detect the growth a specialized fluorescence microscopy technique was applied, the so-called total internal reflection fluorescence microscopy

\footnotetext{
*jfb@elektro.dtu.dk

†mhjensen@nbi.dk
}

(TIRFM). This technique utilizes total internal reflection to create an evanescent electromagnetic field adjacent to the glass slide, thereby exclusively exciting fluorophores in only a very thin volume. The penetration depth, $d$, depends in a specific manner on the wavelength and the angle of the incident light, as well as the refractive indices of the media [17]. In the setup, an argon laser was used along with a fused silica slide in contact with water, leading to a penetration depth of $d=150 \mathrm{~nm}$. The TIRFM images of the fibrillation process were obtained at initial glucagon concentration of $\rho=0.25 \mathrm{mg} / \mathrm{ml}$ in aqueous buffer $(50 \mathrm{mM}$ glycine $\mathrm{HCl} \mathrm{pH}$ $2.5)$ with preformed seeds. Images of the growth are shown in Fig. 1 at three consecutive times $t=0,216,407 \mathrm{~min}$ (see [11] for details on the experiment).

Because the fibrils grow along the glass slide we are able to track each fibril length as function of time. We monitor 16 independent fibrils for each image frame in the experiment. The time interval, $\Delta t$, between frames varies from a minimum value of $\Delta t_{\min }=1 \mathrm{~min}$ to a maximum value $\Delta t_{\max }=35 \mathrm{~min}$, with a typical value of $\Delta t=10 \mathrm{~min}$. The total duration of the experiment is $t=525 \mathrm{~min}$. The combined results for the 16 fibrils are shown in Fig. 2.

A striking feature of the fibril dynamics is its discrete nature, where long periods of growth are interrupted by extended periods of stasis (stop state). This prompted us to collect the statistics of time spent in the growth $(g)$ and the stop $(s)$ state, $f_{g}(t)$ and $f_{s}(t)$, respectively, for all 16 fibrils. In Fig. 3, these distributions are shown on semilogarithmic plots. Note that the finite sampling rate implies that actual time spent in given state can only be estimated in terms of upper and lower bounds [18]. The upper estimates are shown by the dashed blue curves and the lower estimates are shown by the full blue curves. As seen in the figure, the difference between the distributions for the upper and lower estimates of both $f_{g}$ and $f_{s}$ are marginal. All distributions are very well fitted by exponential functions, $f_{g}(t) \sim \exp \left(-k_{-} t\right)$ and 

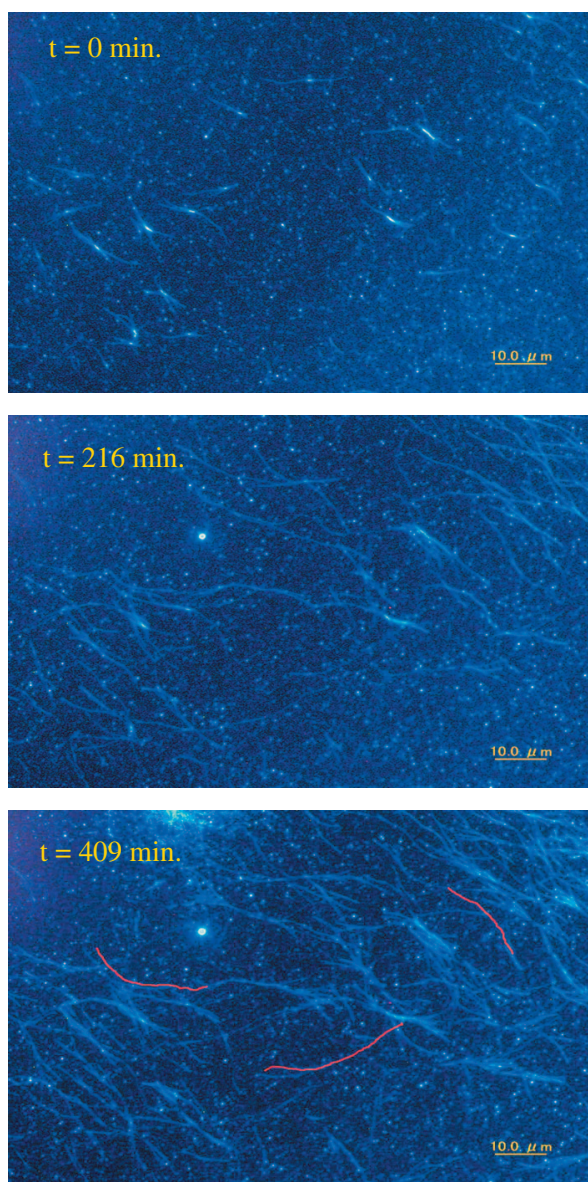

FIG. 1. (Color) TIRFM images of glucacon fibril growth with initial glucagon concentration of $\rho=0.25 \mathrm{mg} / \mathrm{ml}$ in aqueous buffer (50 $\mathrm{mM}$ glycine $\mathrm{HCl}, p \mathrm{H} \mathrm{2.5)}$ at three consecutive times after the initiation of the aggregation. Red lines in the last picture mark examples of fibrils which are tracked during the growth process.

$f_{s}(t) \sim \exp \left(-k_{+} t\right)$, as shown by the yellow curves (here, the dashed yellow curve is the fit to the upper estimates and the full yellow curve is the fit of the lower estimates). The fits are of excellent quality over almost two decades, as signified by high $R$ values $\left(R^{2}>0.98\right)$. The values obtained are

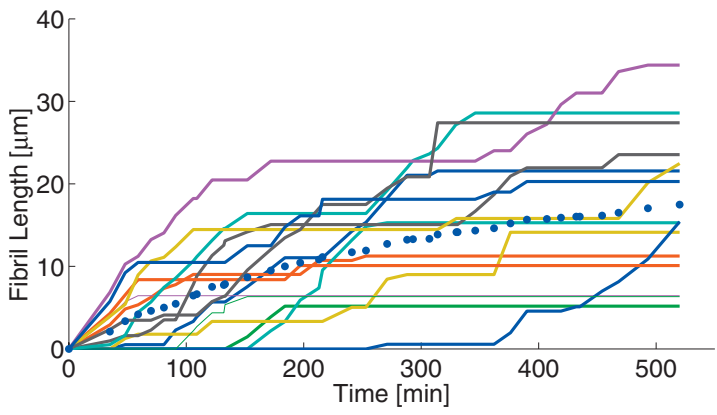

FIG. 2. (Color) Length as a function of time for 16 fibrils tracked from the images shown in Fig. 1. Note the long plateaus, corresponding to the stop states, followed by shorter (on average) growing periods. The average growth is indicated with a dotted blue line. The sampling time between each image can be seen from the time separation between each point.
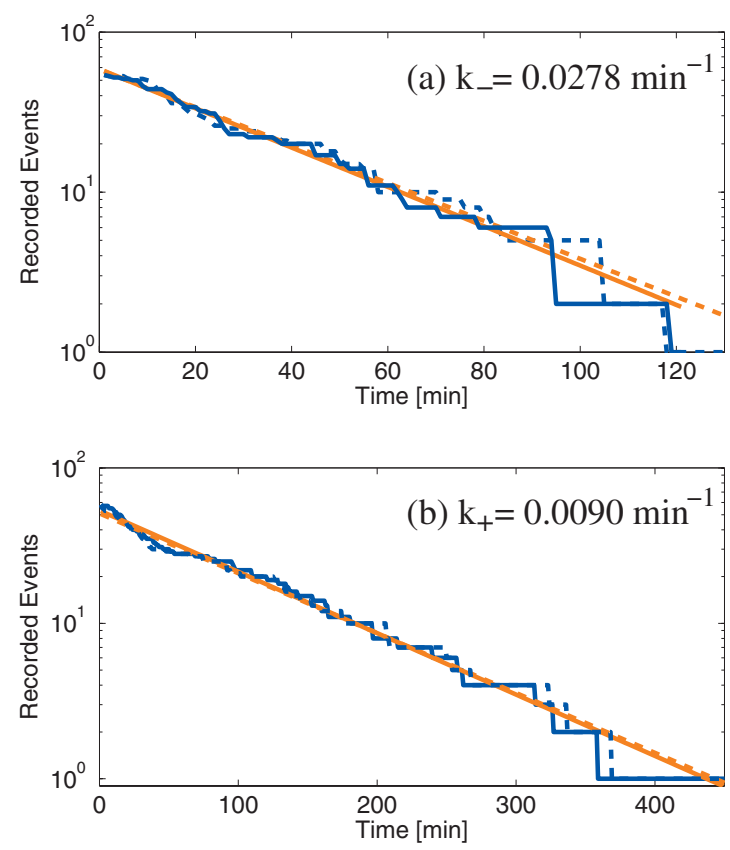

FIG. 3. (Color) (a) Growth $f_{g}(t)$ and (b) stop $f_{s}(t)$ times distributions on semilogarithmic scales. Blue lines are data and yellow straight lines are the exponential fit. Both for data and fits, continuous lines are the lower estimates and dashed lines are the upper estimates (see text). All fits are of extremely good quality as indicated by the large $R$ value, $R^{2}>0.98$.

$k_{+}=9.0 \times 10^{-3} \mathrm{~min}^{-1}$ and $k_{-}=2.8 \times 10^{-2} \mathrm{~min}^{-1}$. A series of four independent experiments $\mathrm{A}-\mathrm{D}$ have been performed with the same glucagon monomer concentration $(0.25 \mathrm{mg} /$ $\mathrm{ml})$ and $\mathrm{pH}$ (2.5) with differences in the seed concentrations $(\sim 20 \%$ variation) and data acquisition only. Specifically, the maximal frame length was varied by a factor 3 and the minimum frame length by a factor 18 . In all four cases the same analysis and procedure was performed resulting in exponential distributions of similar high quality. The results are summarized in Table I.

The observed stop-and-go behavior of fibril dynamics is clearly not associated with the discrete nature of monomer attachment [19]. The simplest model of the process is to assume that the fibril exhibits two internal states: one in which it is allowed to grow, with a rate $g$, and one in which it cannot grow. The intrinsic transition rates between the two states is then identified with the observed transition rates $k_{+}$ (stop $\rightarrow$ growth) and $k_{-}$(growth $\rightarrow$ stop) [see Fig. 4(a)].

The Markovian nature of the model implies, that the time spent in each state is exponentially distributed which is indeed consistent with the results from the data analysis [Fig. $3]$, leading to estimates of $k_{+}$and $k_{-}$. Denoting the total rate $k=k_{+}+k_{-}$, one can calculate the probability of being in the growing state, $p_{+}=k_{+} / k$ and in the stopped state, $p_{-}=k_{-} / k$. Table I presents a summary of the parameters in four different experiments. By comparing the four different series, we observe that the rates $k_{+}$and $k_{-}$vary quite significantly, up to a factor 3 . A possible explanation for this could be the variation in sampling frequency (as is indicated in Table I) due to heating of the sample by the laser. Indeed, the dependency of the fibril growth on both the laser intensity as well as the 
TABLE I. Estimated kinetic parameters (rows 1-5) and parameters for the data acquisition (rows 6-9) in four different experiments, A-D. FL stands for frame length. All figures of this Rapid Communication refer to the experiment of the first column, experiment A. The average growth rate of the fibrils over the total time of each experiment is indicated by $g$ (no appreciable effect of depletion is observed). These values are comparable to the measurements in [11].

\begin{tabular}{lcccc}
\hline \hline Parameters & Expt. A & Expt. B & Expt. C & Expt. D \\
\hline$k_{+}\left(\mathrm{min}^{-1}\right)$ & $0.0090 \pm 0.0001$ & $0.0192 \pm 0.0004$ & $0.0075 \pm 0.0001$ & $0.0059 \pm 0.0001$ \\
$k_{-}\left(\mathrm{min}^{-1}\right)$ & $0.0278 \pm 0.0005$ & $0.04780 \pm 0.00002$ & $0.023 \pm 0.002$ & $0.0166 \pm 0.0002$ \\
$p_{+}$ & 0.244 & 0.249 & 0.247 & 0.260 \\
$p_{-}$ & 0.756 & 0.751 & 0.753 & 0.740 \\
$g(\mathrm{~nm} / \mathrm{min})$ & 135 & 116 & 111 & 56 \\
Tot time $(\min )$ & 525 & 340 & 630 & 1030 \\
Max FL(min) & 35 & 27 & 24 & 90 \\
Min FL (min) & 1 & 3 & 1 & 18 \\
Av FL (min) & 13.7 & 11.8 & 20.7 & 30.9 \\
\hline \hline
\end{tabular}

illumination time has been observed under similar experimental conditions for $\beta_{2}$-microglobulin kinetics [20].

The striking result is that, even though both transition rates vary among experiments, they combine in such a way that the probabilities of growing and stopping, $p_{+}$and $p_{-}$, do not change appreciably in different experiments. In particular, $p_{+}$is always very close to $1 / 4$ and consequently $p_{-}$is very close to $3 / 4$. One may wonder whether this ratio is really independent of the monomer concentration, as in the model. Due to experimental limitations, this has not been directly tested. However, the observed average growth rate,

(a)

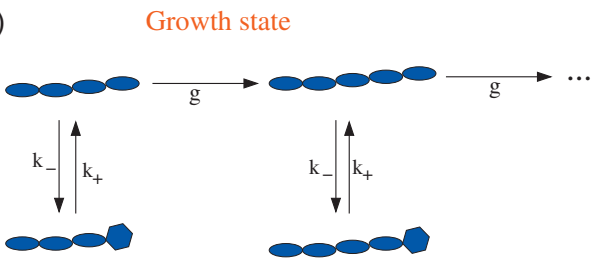

Stop state

(b)

Growth state

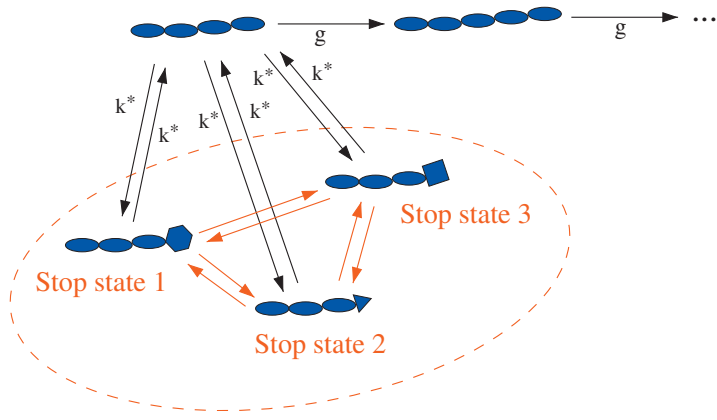

FIG. 4. (Color) (a) The simplest two-state model of fibril growth. The fibril is assumed to be in one of two states with intrinsic transition rates of $k_{+}$and $k_{-}$. In the growing state, monomer attachment occurs with the rate $g$. (b) The iso-energetic four state model where a fixed transition rate, $k^{*}=k_{+}=k_{-} / 3$, between growth and stop states is assumed. The intrinsic rates between the different stop states (red arrows) are irrelevant to the observed kinetics. $g$, displays more than a twofold variation from experiment A to experiment $\mathrm{D}$, suggesting notable differences in the local monomer concentration of the growing fibrils, or the effect of laser heating as discussed earlier. If the state probabilities were sensitive to changes in the concentration, we would have expected a correlation between $p_{ \pm}$and $g$. We further tried to test the pertinence of the state probabilities by dividing the data sets into two parts, one corresponding to the first half of the experiment and the other to the second half, and measuring $p_{+}$and $p_{-}$separately in the two parts. Unfortunately, the statistical noise increased significantly when considering subparts of the data series and neither added to nor altered our conclusions.

The interpretation of our results is that the variation in experimental conditions affects the activation barrier between the stop and grow states, without affecting their freeenergy difference. Understanding the origin of this scenario would require the exact decomposition of this free-energy difference into its energetic and entropic contributions. In this perspective, the measured constancy of $p_{ \pm}$suggests that the free-energy difference between the two states is predominantly entropic. This explanation is the most immediate since it does not require a specific physical mechanism acting on the activation barrier only (without affecting the energy of the two states). Assuming the energy difference to be negligible, the ratio $p_{-} / p_{+} \approx 3$ then reflects the presence of three stop configurations for each growing one, all of them being isoenergetic. This model is illustrated in Fig. 4(b), where all individual transition rates, $k^{*}$, are set equal to the observed transition rate to the growth state, i.e., $k^{*}=k_{+}=k_{-} / 3$. Note that in this picture the internal transition rates between the stop states, indicated by red arrows in Fig. 4(b), are irrelevant for the observed stop-and-go kinetics [21].

To conclude, we have presented a stop-go model for glucagon fibril elongation. In fact, similar kinetic behavior has recently been reported for the fibril elongation of $A \beta$ peptides [22,23] as well as for $\alpha$ synuclein [24] although the time scales involved are 1-2 orders of magnitude faster. This suggests that the observed stop-go kinetics reflects the presence of some kind of structural change at the fibril ends, 
which is not necessarily specific to glucagon. In this scenario, the protein properties would affect the barrier height and thus the time scale of the process, only. The ultimate goal would be to test our prediction against simulations and experiments addressing the molecular aspects of the assembly process, which at present stage is poorly understood [22,25,26].

If our hypothesis about the existence of approximately isoenergetic states holds up to scrutiny, it suggests that there may be an additional dimension in the fibrillation energy landscape that cannot easily be identified by conventional techniques. Glucagon is known to adopt a number of different conformations depending on the fibrillation conditions, but these conformations differ considerably in energy and are unlikely to coexist to an equal extent [27]. Rather, it is possible that we have a number of closely related states with different propagation properties which are separated by high local activation barriers within a relatively flat ground-state level. Specific configurational peptide models with a multitude of approximately isoenergetics states were proposed in [28]. Here, however, these states are considered as obligatory intermediates in the fibrilation process implying a different dynamical mechanism. Further experimental studies are required to establish the validity of our scenario.

This work was supported by the Villum Kann Rasmussen Foundation, Lundbeck Foundation, and the Danish National Research Foundations. We are grateful to Christian Rischel and Joachim Mathiesen for discussions at the early stage of this work.
[1] C. M. Dobson, Nature (London) 426, 884 (2003).

[2] F. Chiti and C. M. Dobson, Annu. Rev. Biochem. 75, 333 (2006).

[3] M. R. Sawaya et al., Nature (London) 447, 453 (2007).

[4] T. P. J. Knowles, J. F. Smith, A. Craig, C. M. Dobson, and M. E. Welland, Phys. Rev. Lett. 96, 238301 (2006).

[5] T. P. J. Knowles, J. F. Smith, G. L. Devil, C. M. Dobson, and M. E. Welland, Nanotechnology 18, 044031 (2007).

[6] S. Chen, F. A. Ferrone, and R. Wetzel, Proc. Natl. Acad. Sci. U.S.A. 99, 11884 (2002).

[7] F. Ferrone, in Methods in Enzymology, edited by R. Wetzel (Elsevier Academic Press, San Diego, 1999), p. 256.

[8] F. Librizzi and C. Rischel, Protein Sci. 14, 3129 (2005).

[9] S. B. Padrick and A. D. Miranker, Biochemistry 41, 4694 (2002).

[10] V. Foderà, D. Librizzi, M. Groenning, M. van de Weert, and M. Leone, J. Phys. Chem. 112, 3853 (2008).

[11] C. B. Andersen, H. Yagi, M. Manno, V. Martorana, T. Ban, G. Christiansen, D. Otzen, Y. Goto, and C. Rischel, Biophys. J. 96, 1529 (2009).

[12] A. S. P. Svane, K. Jahn, T. Deva, A. Malmendal, D. Otzen, J. Dittmer, and N. C. Nielsen, Biophys. J. 95, 366 (2008).

[13] P. A. Christensen, J. S. Pedersen, G. Christensen, and D. E. Otzen, FEBS Lett. 582, 1341 (2008).

[14] C. L. P. Oliveira, M. A. Behrens, J. S. Pedersen, K. Erlacher, D. Otzen, and J. S. Pedersen, J. Mol. Biol. 387, 147 (2009).

[15] J. S. Pedersen, D. Dikov, and D. Otzen, Biochemistry 45, 14503 (2006).

[16] C. B. Andersen, D. Otzen, G. Christiansen, and C. Rischel, Biochemistry 46, 7314 (2007).

[17] T. Wazawa and M. Ueda, Advances in Biochemical Engineering/Biotechnology (Springer, Berlin, 2005), p. 77.

[18] The finite sampling frequency implies that the exact time of a state transition (growth $\leftrightarrow$ stop) can be anywhere in the interval between two observations. Therefore, an upper and lower bound of the time spent in a particular state is simple given by choosing either the "outer" or the "inner" observation points.
[19] Assuming a cylindrical shape of the fibril the monomer attachment rate, $r$, is given by $r \approx g \pi R^{2} / V_{g l u} \approx 10^{3} \mathrm{~min}^{-1}$, where $g \approx 100 \mathrm{~nm} / \mathrm{min}$ is the average observed elongation rate (see table I), $R \approx 5 \mathrm{~nm}$ is the fibril radius, and $V_{g l u} \approx 4 \mathrm{~nm}^{3}$ is the volume of a fibrillated glucagon peptide. This rate is several orders of magnitude larger than the observed transition rates.

[20] D. Ozawa, H. Yagi, T. Ban, A. Kameda, T. Kawakami, H Naiki, and Y. Goto, J. Biol. Chem. 284, 1009 (2008).

[21] The irrelevance of the transition rates between the different stop states is easily demonstrated from the kinetic equation $d p_{i}(t) / d t=\sum_{j} k_{i j} p_{j}(t)$, where $p_{i}(t)$ is the probability for the fibril to be in state $i$ at time $t, k_{i j}$ is the rate for the transition $j \rightarrow i$, and the sum is over all four possible states $(j=1,2,3$ for the stop states and $j=4$ for the growth state). Here, the diagonal elements $k_{i i}$ is defined from conservation of probability, $k_{i i}=-\sum_{j \neq i} k_{j i}$. The time distribution for the stop mode, $p_{-}(t)$ $=\sum_{j=1}^{3} p_{j}(t)$, is obtained by treating the growth state as an absorbing state $\left(k_{j 4}=0\right.$, for $\left.j \neq 4\right)$, leading to $d p_{-}(t) / d t$ $=-\sum_{j=1}^{3} k_{4 j} p_{j}(t)$. Using the initial condition, $p_{-}(0)=1$ and the model assumption that the rate of transition from each of the stop states to the growth state is identical, $k_{4 j}=k^{*}$, the solution to this equation becomes $p_{-}(t)=\exp \left(-k^{*} t\right)$, from where we identify $k_{+}=k^{*}$. Applying the same line of arguments for the growth state shows that $k_{-}=3 k^{*}$.

[22] M. S. Z. Kellermayer, A. D. Karsai, M. Benke, K. Soo, and B. Penke, Proc. Natl. Acad. Sci. U.S.A. 105, 141 (2008).

[23] B. Tadato, M. Hoshino, S. Takahashi, D. Hamada, K. Hasegawa, H. Naiki, and Y. Goto, J. Mol. Biol. 344, 757 (2004).

[24] W. Hoyer, D. Cherny, V. Subramaniam, and T. M. Jovin, J. Mol. Biol. 340, 127 (2004).

[25] W.-F. Xue, S. W. Homans, and S. E. Radford, Proc. Natl. Acad. Sci. U.S.A. 105, 8926 (2008).

[26] D. J. Selkoe, Nature (London) 426, 900 (2003).

[27] J. S. Pedersen and D. Otzen, Protein Sci. 17, 2 (2008).

[28] C. F. Lee, J. Loken, L. Jean, and D. J. Vaux, Phys. Rev. E 80, 041906 (2009). 